\title{
Construcción de un Simulador Dinámico para Procesos de Cristalización por Lotes a Vacío
}

\author{
Jesús Carrillo ${ }^{1}$, Miguel V. Teco ${ }^{2 *}$, Anselmo Osorio ${ }^{2}$ y Eusebio Bolaños ${ }^{1}$ \\ (1) Instituto Tecnológico de Orizaba, División de Estudios de Posgrado e Investigación, \\ Av. Oriente 9 No. 852, 94320 Orizaba, Ver.-México (e-mail: ane18@latinmail.com). \\ (2) Universidad Veracruzana, Facultad de Ciencias Químicas, Prolongación Oriente 6 No.1009, \\ 94340 Orizaba, Ver.-México (e-mail: anselmo_osorio@yahoo.com; mteco@hotmail.com).
}

\section{Resumen}

Se presenta un simulador dinámico para procesos de cristalización por lotes a vacío utilizando un modelo no lineal El modelo se basa en la ecuación de balance de población, balances de materia y energía para el interior del cristalizador, la ecuación de continuidad del vapor en la camisa de calentamiento y relaciones constitutivas. Utilizando la técnica de momentos de distribución de tamaño de cristal, la ecuación de balance de población se convierte en ecuaciones diferenciales. La construcción del simulador se realizó utilizando Visual Basic. Se propone un ejemplo y se comparan los resultados con otro simulador y con datos experimentales de un equipo piloto, obteniéndose resultados concordantes.

Palabras claves: cristalización, lotes a vacío, simulación de procesos, modelo matemático

\section{Dynamic Simulator Construction for Vacuum Batch Crystallization Processes}

\begin{abstract}
A dynamic simulator for vacuum batch crystallization processes using a non-linear model is presented. The model is based on the equation of balance population, on mass and energy balances inside the crystallizer, on the steam continuity equation for the heating jacket and on constitutive relations. Using the technique of crystal size distribution moments, the equation of balance population is converted into a set of differential equations. The construction of simulator was done using Visual Basic. An example of its use is proposed comparing results with those of another simulator and with experimental information obtained in a pilot equipment pilot, obtaining similar results.
\end{abstract}

Keywords: crystallization, vacuum batch, process simulation, mathematical modeling 


\section{INTRODUCCIÓN}

La cristalización es un proceso de purificación y separación de un soluto a partir de una solución sobresaturada, mediante la formación de cristales en el seno de la solución, logrando purezas superiores al 99\%. Posee gran importancia económica en la industria química, ya que requiere mucho menos energía para la separación de lo que se utiliza en la destilación y otros métodos de purificación comunes; además se puede realizar a temperaturas relativamente bajas y a una escala que varía desde unos cuantos gramos hasta miles de toneladas, mejora la apariencia, y hace más fácil el transporte del sólido (Perry, 2003).

La cristalización se utiliza en la producción de azúcar, fertilizantes y productos farmacéuticos, entre otras industrias. El objetivo de la cristalización a nivel industrial es que el producto tenga una distribución de tamaño de cristal (DTC) y pureza específicas, en lugar de un producto aleatorio (Ulrich, 2003).

El incremento de la calidad en la industria de procesos y la minimización de costos de producción requiere de la comprensión de cómo obtener una DTC apropiada. El mejoramiento de métodos de medición (Martins et al., 2005a, 2005b) y la capacidad de procesamiento computacional actual ofrecen la posibilidad de extender las simulaciones en computadora a este campo. Un proceso se desea modelar de acuerdo a los fenómenos químicos y físicos presentes y no de acuerdo a las restricciones computacionales (Wulkow et al., 2001). Pero conforme las ecuaciones del modelo describen ampliamente estos fenómenos (nucleación primaria y secundaria, crecimiento de cristal, mecanismos de aglomeración y atrición, etc.), la solución numérica es un obstáculo a su aplicación práctica (Vollmer y Raisch, 2002).

Se han propuesto diversas soluciones al modelo del proceso de cristalización, implementadas en software específico especialmente adecuado para unidades de proceso que son modeladas por ecuaciones de balance de población. En Wulkow et al. (2001), el algoritmo numérico y la interfase de usuario se implementaron usando el concepto orientado a objetos en el software de simulación Parsival. Sin embargo, para propósitos de control es necesario tener un modelo suficientemente simple, tal que el problema de la síntesis de controladores sea tratable y cuyo comportamiento dinámico se aproxime, en algún sentido, al modelo detallado y por lo tanto también a la planta real. Es deseable mantener, tanto como sea posible, la estructura del modelo original para preservar la interpretación física de los estados del sistema y la naturaleza distribuida del mismo (Vollmer y Raisch, 2002). En Laloue et al. (2007), se resuelve el modelo completo de la cristalización usando un método de diferencias finitas para la discretización de la variable tamaño de cristal, mientras que en Yang et al. (2007), se discute la aproximación de modelos mecanísticos complejos con modelos estadísticos a través de procedimientos de optimización iterativos que hacen uso de un modelo de proceso Gaussiano para aproximar localmente el modelo complejo. Con el gran desarrollo actual del software, procesos físicos complejos se pueden modelar sin mayores problemas.

Los sistemas por lote son convenientes y útiles en el laboratorio y en la línea de producción. En la industria se usan para volúmenes bajos aislados y productos de alto valor agregado, además son valiosos por su flexibilidad. Considerando estas características, el objetivo de este trabajo es mostrar que a partir de la solución de la ecuación de balance de población utilizando el método de momentos, es posible construir un simulador para describir la DTC de un sistema de cristalización industrial. La operación del sistema contempla la implementación de perfiles dinámicos de regulación de vacío como una técnica de operación nueva en cristalización por lotes. Esto permite manipular la sobresaturación promedio, evita la aglomeración del producto cristalino formado y elimina el uso de licor no saturado como medio de disolución durante la operación del lote (Gerstlauer et al., 2002).

\section{MODELO MATEMATICO DE LA CRISTALIZACIÓN POR LOTES A VACÍO}

El modelo teórico del proceso de cristalización por lotes a vacío involucra al balance de población, en el que se define la distribución del tamaño del cristal (DTC), los balances de masa y energía, así como las relaciones constitutivas (Mullin, 2001) para los fenómenos de nucleación y crecimiento de cristales. El modelo consiste en una serie de ecuaciones integro-diferenciales no lineales; en las que 
se consideran aspectos como la nucleación primaria, el crecimiento del cristal, la ruptura de cristales debido a las colisiones cristal-cristal o cristal-agitador y el balance de población (escala microscópica).

La solución del modelo matemático de la cristalización por lotes a vacío que se utiliza en este simulador, se realiza con el método de momentos de distribución del tamaño de cristal, el cual, asume que el crecimiento de los cristales es independiente del tamaño y de la muerte de los mismos, con esta técnica se obtiene un conjunto de cuatro ecuaciones diferenciales que describen la distribución del tamaño del cristal.

En la escala macroscópica, se tiene el modelo general de toda la operación unitaria de cristalización con los balances de materia y energía, en el interior y en la camisa de enfriamiento (Gerstlauer et al., 2002). Por la cantidad de ecuaciones que utiliza el modelo matemático de la cristalización por lotes a vacío, se propone la construcción de un simulador dinámico, que proporcione resultados satisfactorios durante el tiempo de simulación.

El equipo piloto disponible para la obtención de datos experimentales reales de proceso es un cristalizador por lotes de acero inoxidable de $12.8 \mathrm{~L}$, con chaqueta de calentamiento de $11.1 \mathrm{~L}$. de capacidad, 4 deflectores de $17.0 \mathrm{~cm}$ de ancho por $3.5 \mathrm{~cm}$ de largo, flecha de agitación de $39.0 \mathrm{~cm}$ de largo, bomba de vacío, caldera y motor de agitación con variador de velocidad.

\section{SIMULADOR NUMÉRICO PARA LA CRISTALIZACION POR LOTES A VACIO}

El comportamiento de muchos procesos fisicoquímicos en ingeniería química, particularmente aquellos que experimentan cambios dependientes del tiempo (transitorios), se modelan a través de un sistema de ecuaciones diferenciales ordinarias (EDO). Si bien muchas EDO importantes se pueden resolver a través de técnicas analíticas bien conocidas, un gran número de ecuaciones diferenciales físicamente significativas no pueden resolverse de esta forma.

Los algoritmos explícitos de resolución de EDO's implican la evaluación explícita de las derivadas y el avance paso a paso en el tiempo sin necesidad de recurrir a procedimientos iterativos. Existen dos métodos muy difundidos y fáciles de usar que utilizan arranque automático. Estos son: el método de Euler y los métodos Runge-Kutta (4to. y 5to. orden)

Para la solución del modelo matemático se empleo el método de Runge-Kutta. Una variante es el llamado Runge-Kutta-Fehlberg (denotado como RKF45), que aplica los dos metodos Runge-Kutta de 4to. y 5to. orden para determinar si el tamaño de paso de cálculo es el apropiado. En cada paso de integración, se realizan y se comparan dos aproximaciones diferentes para la solución. Si las dos respuestas están dentro de un intervalo conveniente, la aproximación es aceptada; si no están de acuerdo a la precisión especificada, el tamaño de paso es reducido. Si la respuesta necesita mas cifras significativas el tamaño de paso es incrementado.

El simulador dinámico del proceso de cristalización resuelve el modelo matemático de la cristalización por lotes a vacío presentado en Carrillo (2006). Se denomina MoVac 1.0, el cual tiene características tales como: una interfaz amigable con el usuario, la posibilidad de ingresar variables del proceso, y presentar resultados en forma de tablas, gráficas y reportes para una mejor manipulación de los mismos.

Para el diseño y codificación del simulador se utilizó un lenguaje de programación, con el cual se codificaron todas las rutinas, tomando como base el algoritmo numérico Runge-Kutta-Fehlberg, para resolver el modelo matemático conformado por ecuaciones diferenciales. Una vez validado el modelo, se procedió a crear las rutinas para las interfases de configuración, introducción de datos y parámetros específicos del modelo, posteriormente se crearon las interfases de presentación de resultados. Los algoritmos se basan en la programación orientada a objetos (POO) y programación orientada a eventos que proporciona el lenguaje de programación Visual Basic. 
Tabla 1: Resumen del modelo matemático del cristalizador (Carrillo, 2006).

\begin{tabular}{|c|c|c|c|}
\hline BALANCE & \multicolumn{3}{|c|}{ ECUACION REPRESENTATIVA } \\
\hline \multirow{6}{*}{$\begin{array}{l}\text { Ecuación del } \\
\text { balance de } \\
\text { población } \\
\text { (EBP) }\end{array}$} & \multicolumn{3}{|c|}{$\frac{\partial n}{\partial t}+\frac{\partial(G n)}{\partial L}+\frac{n}{V} \frac{\partial V}{\partial t}+D(L)-B(L)+\sum_{k} \frac{n_{k} Q_{k}}{V}=0$} \\
\hline & \multicolumn{3}{|c|}{$\frac{d \mu_{j}}{d t}=j G \mu_{j-1}+B^{0} O^{j}+j G \ln V \mu_{j-1}$} \\
\hline & \multicolumn{3}{|c|}{ Solución de la EBP utilizando la técnica de momentos de la DTC } \\
\hline & $\frac{d \mu_{0}}{d t}=B^{0}$ & $\frac{d \mu_{1}}{d t}=G \mu_{0}(1+\ln (V))$ & $\frac{d \mu_{2}}{d t}=2 G \mu_{1}(1+\ln (V))$ \\
\hline & $\frac{d \mu_{3}}{d t}=3 G \mu_{2}(1+\ln (V))$ & $\frac{d \mu_{4}}{d t}=4 G \mu_{3}(1+\ln (V))$ & $\frac{d \mu_{5}}{d t}=5 G \mu_{4}(1+\ln (V))$ \\
\hline & $D(m, n)=\frac{\mu_{m}}{\mu_{n}}$ & $S(m, n)=\left(\begin{array}{c}\mu_{m+1} \\
\mu_{n}\end{array}\right)-\left(\begin{array}{c}\mu_{m} \\
\mu_{n}\end{array}\right)^{2}$ & \\
\hline
\end{tabular}

\begin{tabular}{|l|l|l|}
\hline $\begin{array}{l}\text { Balance de } \\
\text { masa }\end{array}$ & $\frac{d(C)}{d t}=-\rho_{c} K_{v} h\left[3 \int_{0}^{\infty} G n L^{2} d L+B^{0} L_{0}^{3}\right]$ & $C(t)=C_{0} \quad$ a $\quad t=0$ \\
\hline
\end{tabular}

\begin{tabular}{|l|l|}
\hline $\begin{array}{l}\text { Balance de } \\
\text { energía para } \\
\text { el interior del } \\
\text { cristalizador }\end{array}$ & $\rho V C p \frac{d T}{d t}=\frac{d P V}{d t} c-H_{e v} F_{e v}-\Delta H_{c} \rho_{c} K_{v} V\left[3 \int_{0}^{\infty} G n L^{2} d L+B^{o} L_{0}{ }^{3}\right]+U_{1} A_{1}\left(T_{j}-T\right)$ \\
\cline { 2 - 3 } & $T(t)=T_{0} \quad$ a $\quad t=0$ \\
\hline
\end{tabular}

\begin{tabular}{|l|l|ll}
\hline $\begin{array}{l}\text { Balance de } \\
\text { masa del } \\
\text { vapor }\end{array}$ & $V_{j} \frac{d \rho_{j}}{d t}=F_{s} \rho_{s}-W_{c}$ & $\rho_{j}(t)=\rho_{j 0} \quad$ a $\quad t=0$ \\
\hline
\end{tabular}

\begin{tabular}{|c|c|c|}
\hline \multirow{3}{*}{$\begin{array}{l}\text { Relaciones } \\
\text { constitutivas }\end{array}$} & $G=K_{g} S r^{g} N_{r}^{q}$ & $S r=\frac{C-C_{\text {sat }}}{C_{s a t}}$ \\
\hline & $B^{o}=K_{b} S r^{b} M_{T}^{j} N_{r}^{p}$ & $M_{T}=\rho_{c} K_{v} \mu_{3}(t)$ \\
\hline & $F T M=\Delta L\left(\frac{n_{i}}{n_{0}}\right) * 1000$ & $\begin{aligned} \alpha(L) & =B_{e}+B_{c}+B_{a}-D(L) \\
& =K_{a} S r^{a} M_{T}{ }^{k} N_{r}{ }_{r}^{r} F T M\end{aligned}$ \\
\hline
\end{tabular}

\section{Descripción del simulador}

A continuación se muestran las pantallas generadas para presentación, acceso de datos generales, parámetros cinéticos y reporte de resultados. En la Figura 1, se describe un cristalizador por lotes a vacío que consta de una cámara de evaporación y un condensador barométrico, también se mencionan las variables involucradas en el proceso que son de interés para el sistema de estudio. Por medio de los botones que se ubican en la parte inferior de la ventana (Figura 1) se remarca la zona del equipo de un determinado color, con la finalidad de que el usuario se familiarice con las variables que constituyen el modelo matemático y específicamente las variables más importantes que se pueden manipular durante la operación de un cristalizador por lotes a vacío.

\section{Ingreso de datos generales}

En la Figura 2, se muestra la interfaz gráfica que muestra las generalidades del proceso, se menciona la sustancia a cristalizar, la densidad del cristal, la temperatura final esperada, el tiempo de simulación y el intervalo de impresión; también las propiedades de la solución, la masa del solvente, 
el volumen de la solución, la capacidad calorífica de la solución, la concentración inicial, la concentración de saturación y el calor de cristalización.

Además, las propiedades del vapor de calentamiento, el perfil de agitación ya sea constante o definido por el usuario y el perfil de vacío (lineal, cúbico). Se tiene la flexibilidad de cambiar los valores numéricos de las variables y parámetros antes mencionados dependiendo del experimento a realizar.

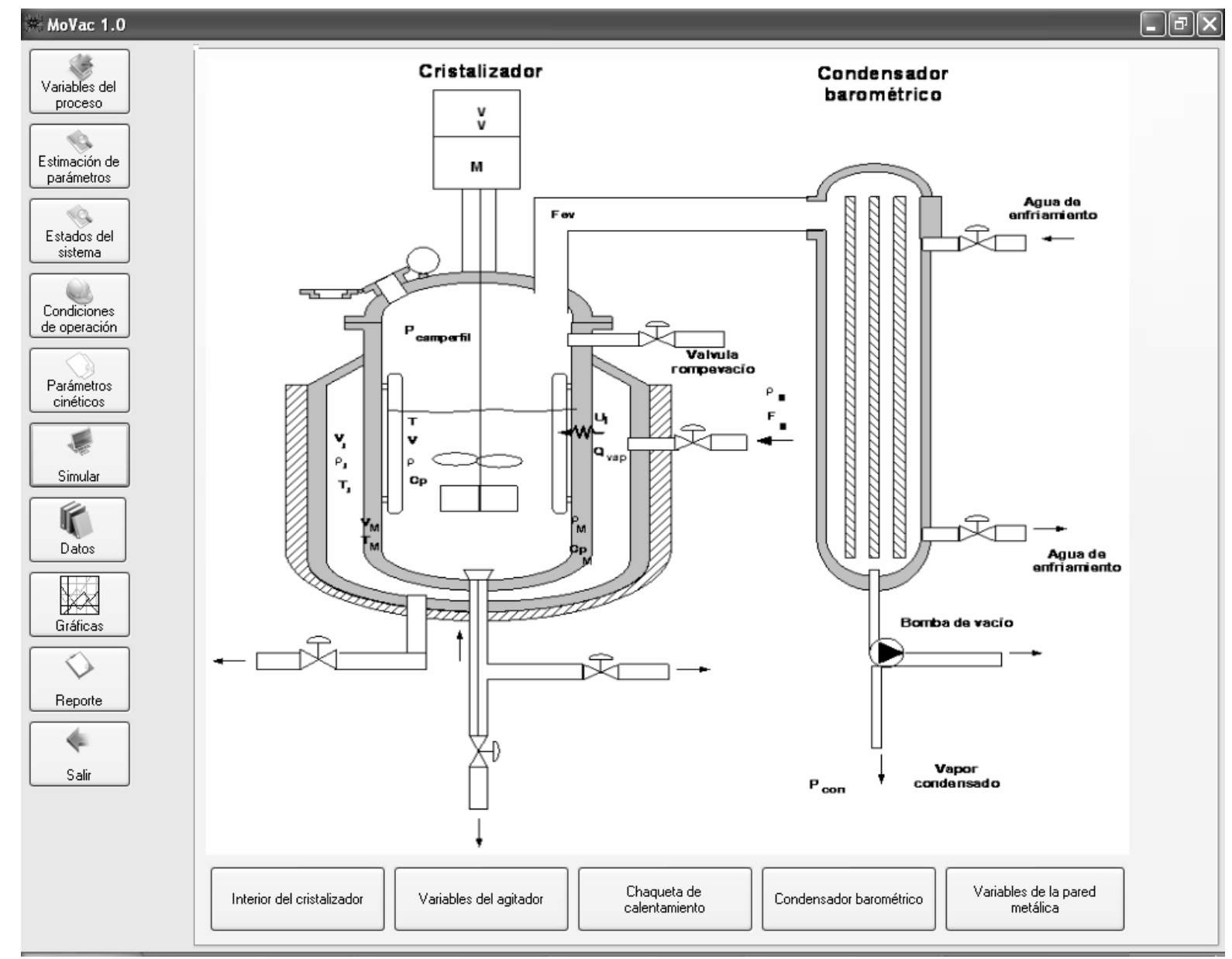

Fig. 1: Cristalizador por lotes a vacío.

\section{Parámetros cinéticos}

Para complementar el modelado del proceso de cristalización, y describir totalmente su comportamiento dinámico, es necesario considerar las relaciones constitutivas de nucleación y crecimiento de cristales. El simulador permite especificar los coeficientes de las ecuaciones de velocidades de nucleación y crecimiento de cristal. Estos coeficientes son específicos de acuerdo a la sustancia que se desea cristalizar o viéndolo desde otro punto de vista corresponden a los coeficientes del soluto en la solución. Estos fenómenos compiten en el cambio de fase desde la solución hacia la fase sólida. En la Figura 3, se ilustra cómo se ingresan los datos de los parámetros cinéticos para la nucleación y el crecimiento de cristales.

\section{Presentación de resultados}

Cuando en las opciones anteriores se han ingresado los valores para las principales variables y parámetros, el simulador MoVac 1.0 resolverá el modelo matemático de la cristalización por lotes a vacío que se propone. Los resultados numéricos se obtuvieron utilizando el método de Runge-KuttaFehlberg, una vez que ha terminado la simulación, se activan tres botones que permiten mostrar los resultados en tres formas distintas: 


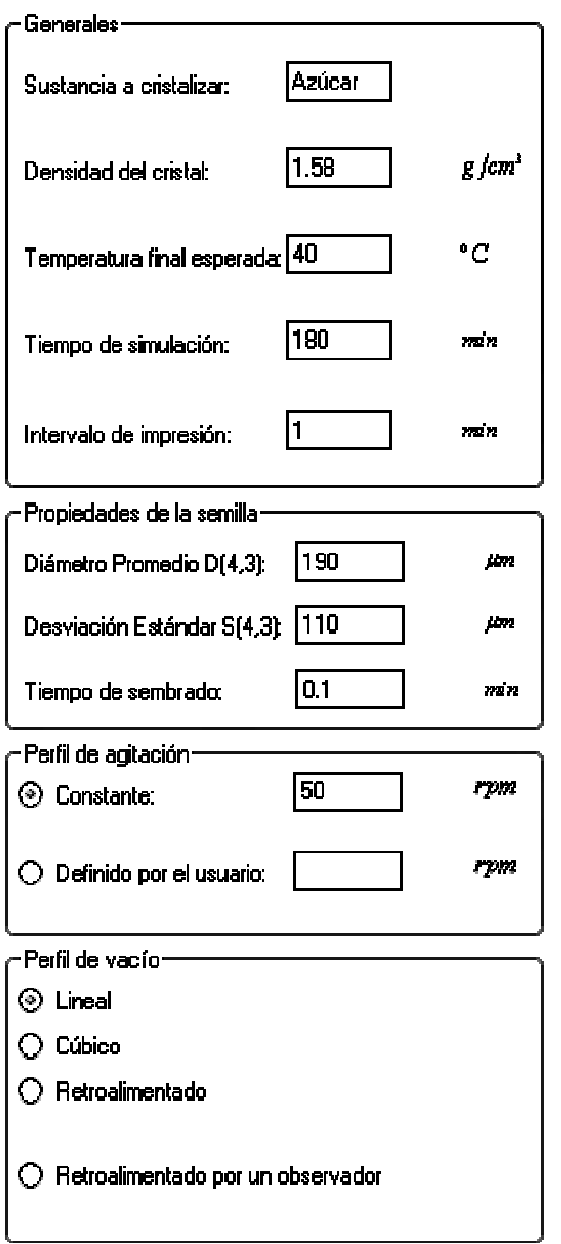

\begin{tabular}{|c|c|c|}
\hline \multicolumn{3}{|l|}{ Propiedades de la solución- } \\
\hline Masa del solverte: & 2922.607 & $g$ \\
\hline Volumen de la solución: & 8543.695 & $m^{2}$ \\
\hline Cp de la solución: & 0.5906 & $\mathrm{cal} / \mathrm{g}^{8} \mathrm{C}$ \\
\hline Concentr ación inicial: & 3.161 & $\frac{g \text { de gazicar }}{g \text { de agua }}$ \\
\hline Concentr ación de saturación & E0.912+0.264*T $T 0.0$ & ${ }^{\circ} \operatorname{Brix}\left(\operatorname{Ten} .^{\circ} \mathrm{C}\right)$ \\
\hline Calor de cristalización: & {$\left[-1.0-\left[0.0655^{n T}\right]\right)^{n}(1000$} & Kcal/moll Temp. ${ }^{\circ} \mathrm{C}$ \\
\hline \multicolumn{3}{|c|}{ Propiedades del vapor de calentamiento- } \\
\hline Cp del vapor: & 1 & $\infty \mathrm{cl} / \mathrm{g}^{\circ} \mathrm{C}$ \\
\hline Densidad del yapor. & 0.0434 & $\mathrm{~g} / \mathrm{cm}^{3}$ \\
\hline Flujp de vapor: & 10 & $c m^{3} / \min$ \\
\hline \multicolumn{3}{|l|}{ Areas de transiferencia de calor. } \\
\hline Del cristafzador: & 2004.464 & $\mathrm{~cm}^{2}$ \\
\hline \multicolumn{3}{|c|}{ Coeficientes de transfierencia de calor } \\
\hline Del interior a la chaqueta: & 0.09418 & $\operatorname{call}\left(\left(\mathrm{cm}^{2}\right)(\mathrm{min})\left({ }^{\circ} \mathrm{C}\right)\right.$ \\
\hline \multicolumn{3}{|l|}{ Volumen de la chaqueta- -} \\
\hline Volumen de la chaqueta: & 11102.9 & $\mathrm{~cm}^{3}$ \\
\hline
\end{tabular}

Fig. 2: Generalidades del proceso.

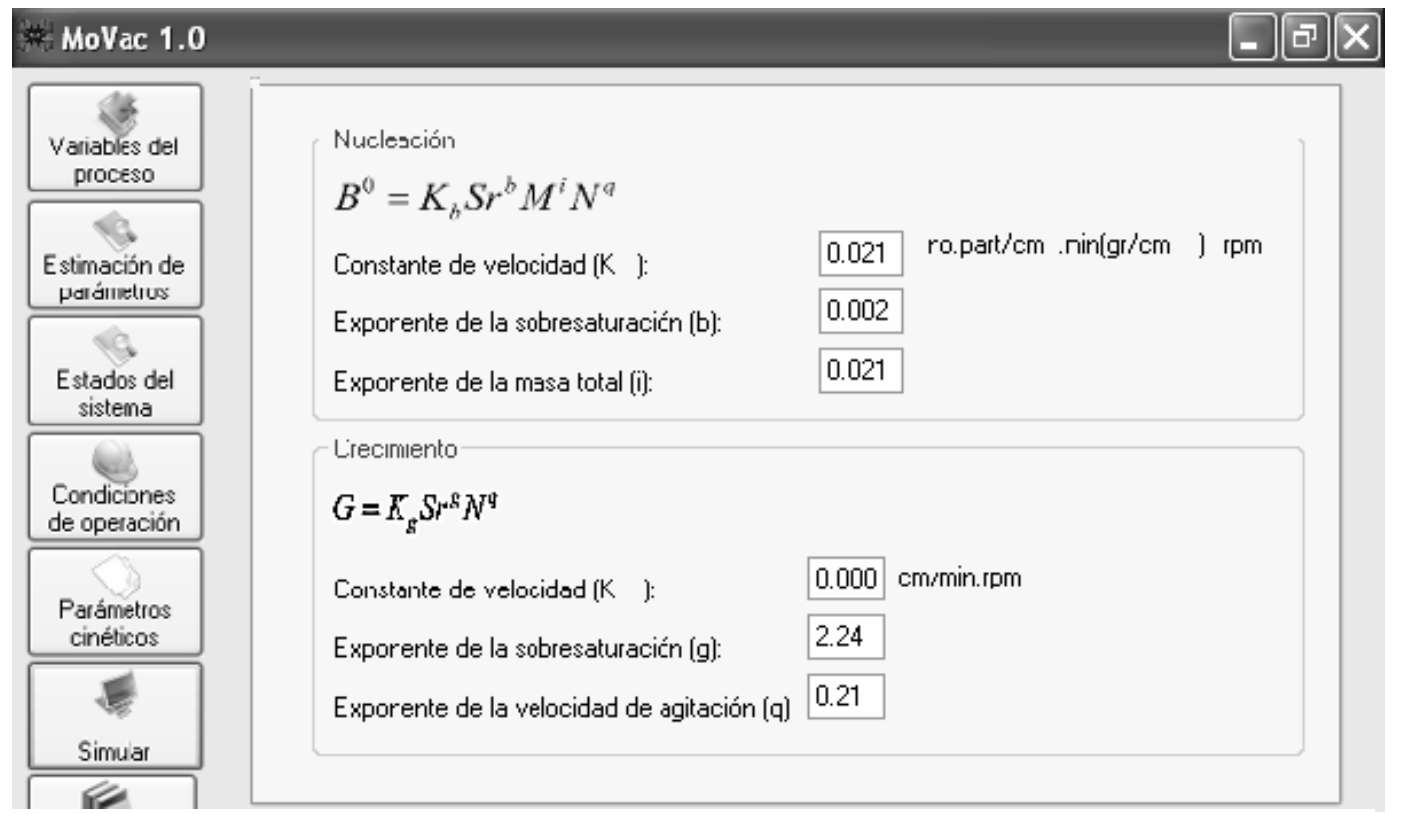

Fig. 3: Parámetros cinéticos

Gráficas (Figura 4), tienen como características importantes que se puede seleccionar y graficar cualquier tipo de variable involucrada en el proceso, hacer acercamientos para visualizar las curvas obtenidas, y exportarlas en algún tipo de imagen electrónica como (PNG, GIF, etc.). Mediante un panel que se encuentra en la parte izquierda de la ventana, se puede visualizar una estructura en forma de árbol que muestra en forma agrupada la totalidad de las variables involucradas en el 
modelo matemático que pueden ser graficadas, teniendo la posibilidad de presentar la evolución en el tiempo de más de una variable a la vez.
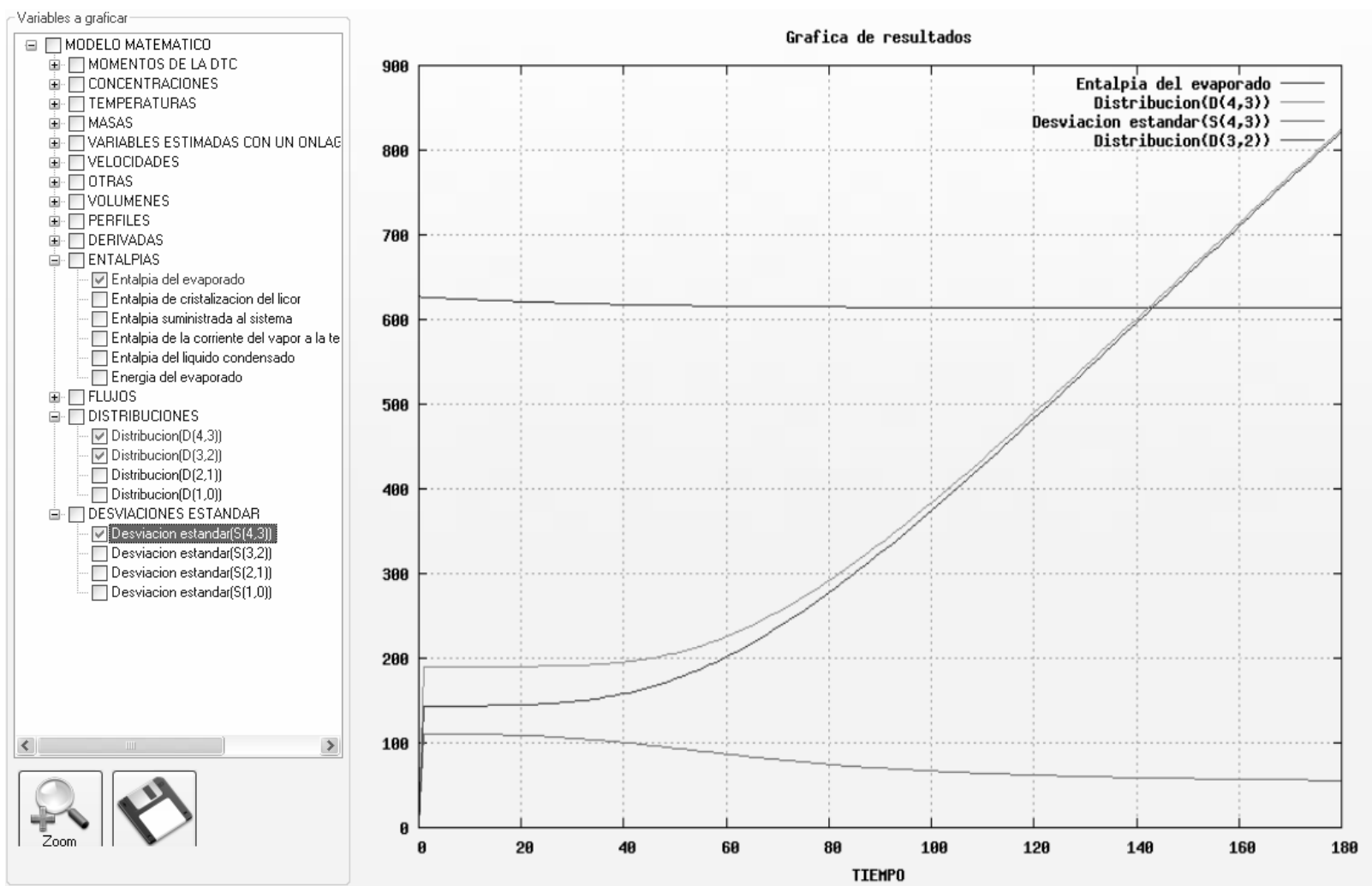

Fig. 4: Gráficas obtenidas por el simulador MoVac 1.0

Tablas (Figura 5), se pueden visualizar los resultados numéricos obtenidos por el simulador, para ser manipulados posteriormente con cualquier tipo de hoja de cálculo (Excel, etc.). Se muestra en forma tabulada la totalidad de las variables de interés involucradas en el proceso de cristalización desde el tiempo cero que considera a las condiciones iniciales de la simulación hasta el tiempo final establecido por el usuario. Todo esto con la finalidad de que se tenga la posibilidad de visualizar en forma numérica los valores obtenidos para ser manejados por algún software de hoja de cálculo y realizar operaciones con ellos.

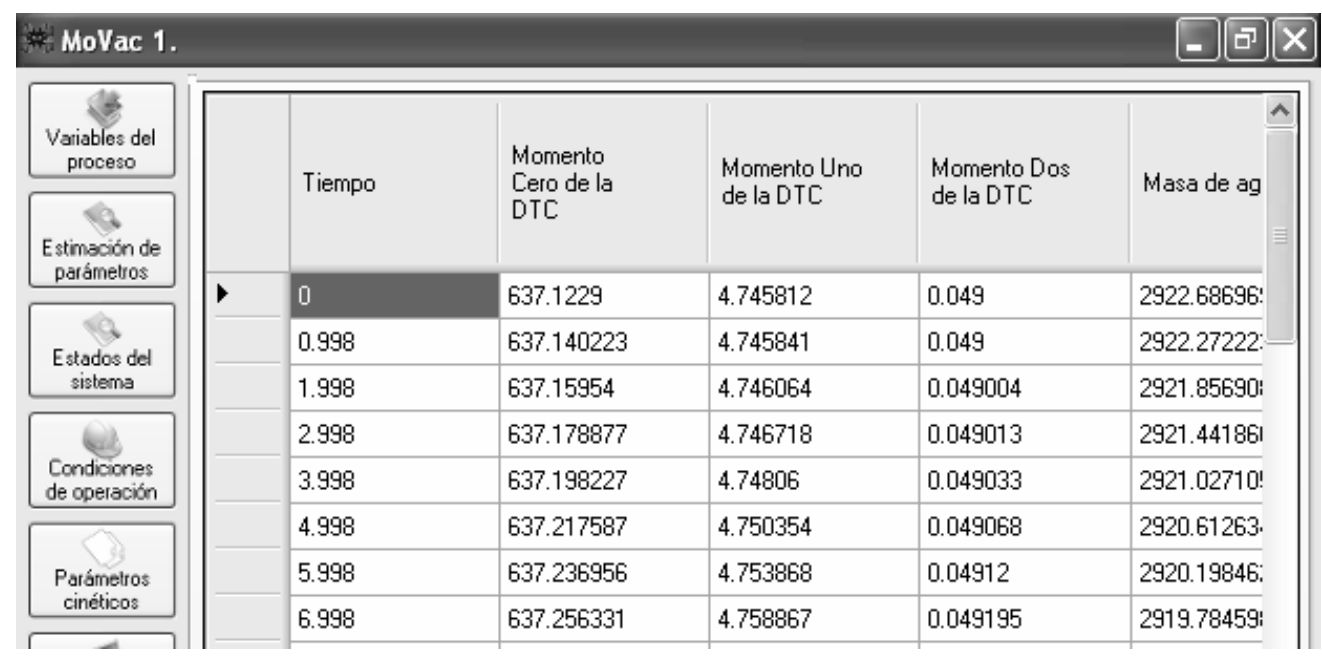

Fig. 5: Tablas obtenidas por el simulador MoVac 1.0

En el Reporte (Figura 6) se muestra el comportamiento numérico de las variables del proceso, principalmente los valores iniciales, finales, valores máximos y mínimos. Está formado por una especie de tabla-resumen, que contiene las ecuaciones diferenciales, ecuaciones algebraicas y 
constantes involucradas en el modelo matemático, presenta los valores inicial, mínimo, máximo y final, en ese orden, de las variables a lo largo de la simulación. Esto con la finalidad de visualizar cuáles fueron los máximos y mínimos (los picos durante la operación) de las variables. Este reporte está hecho mediante un archivo en formato html, de tal forma que se tenga la posibilidad de copiar y pegar para agregarse en un procesador de palabras.

\begin{tabular}{|c|c|c|c|c|c|}
\hline \multicolumn{4}{|c|}{$\begin{array}{l}\text { Reporte de MOVAC } 1.0 \\
\text { Ecuaciones Diferenciales, Algebráicas y Valores constantes }\end{array}$} & \multicolumn{2}{|c|}{ Versión del documento en htm } \\
\hline \multicolumn{6}{|c|}{ Valores calculados para las variables del modelo matemático } \\
\hline & Variables & Valor Inicial & Valor Mínimo & Valor Máximo & Valor Final \\
\hline 1 & Tiempo & 0 & 0 & 179.998 & 180 \\
\hline 2 & Momento Cero de la DTC & 637.1229 & 637.1229 & 640.859066 & 640.859066 \\
\hline 3 & Momento Uno de la DTC & 4.7458125 & 4.7458125 & 52.206079 & 52.206079 \\
\hline 4 & Momento Dos de la DTC & .049 & .049 & 4.273536 & 4.273536 \\
\hline 5 & Momento Tres de la DTC & .0007038 & .0007038 & .351224 & .351224 \\
\hline 6 & Momento Cuatro de la DTC & $1.3375 E-05$ & $1.3375 \mathrm{E}-05$ & .028986 & .028986 \\
\hline 7 & Momento Cinco de la DTC & $3.4 \mathrm{E}-07$ & $3.4 \mathrm{E}-07$ & .002403 & .002403 \\
\hline 8 & Concentracion & 3.161 & 3.161 & 3.161 & 3.161 \\
\hline 9 & Temp. Interior & 70 & 70 & 70 & 39.885268 \\
\hline
\end{tabular}

Fig. 6: Reporte obtenido por el simulador MoVac 1.0

\section{RESULTADOS Y DISCUSIÓN}

El modelo matemático de la cristalización por lotes al vacío, se codificó en el software Microsoft Visual Basic 2005 Express Edition. El simulador está compuesto por tres módulos, principalmente, el módulo de presentación e ingreso de datos de variables de proceso y parámetros, el módulo de cálculo numérico, para construir las subrutinas que permiten la aproximación numérica de las ecuaciones del modelo a partir de las condiciones iniciales establecidas. Y el módulo de resultados, que permite visualizar el resultado de la simulación en tres diferentes formas: Gráficas: que permite ver gráficamente la evolución en el tiempo de cualquier variable involucrada en el modelo matemático. Tablas: muestra en forma de columnas los cálculos obtenidos para cada una de las variables involucradas en la simulación. Y por último Reporte: que permite visualizar en forma de reporte (pagina html) un resumen de todas las variables involucradas en la simulación.

La Figura 7, muestra los resultados numéricos obtenidos por el simulador MoVac 1.0, el simulador CristBatch 2.1 y los resultados obtenidos experimentalmente en el equipo piloto disponible, con operación por enfriamiento $(\bullet)$ y a vacío $(\times)$. El simulador dinámico CristBatch Versión 2.1 permite simular el proceso de cristalización por lotes aplicando técnicas de enfriamiento programado, mezclado mejorado y siembra de cristales. La interfaz gráfica de este simulador fue creada en el compilador Microsoft Visual C++6.0, la cual permite modificar las variables del proceso y presenta los resultados de la simulación en forma tabulada y gráfica. En este simulador se resuelve el modelo matemático por medio de la discretización de la EBP, transformándola en un conjunto de ecuaciones diferenciales ordinarias. La Figura 7(a) muestra el momento de distribución $\mathrm{D}(4,3)$, correspondiente al diámetro promedio de los cristales, se observa que ambos simuladores muestran una tendencia similar a la que siguen los datos experimentales. La Figura 7(b) muestra la desviación estándar (momento $\mathrm{S}(4,3)$ ) con valores similares en ambos simuladores, correlacionándose en forma muy cercana con los datos experimentales durante todo el proceso. También, en la Figura 7(c), se pueden observar las trayectorias de la temperatura en el interior conforme se desarrolla el proceso de cristalización. Los errores relativos de estas variables se muestran en la Figura 8, en donde se puede observar que el comportamiento dinámico del simulador construido proporciona resultados satisfactorios.

Es importante destacar que una de las principales características del simulador es que es posible realizar la estimación de parámetros, no mostrada en este trabajo debido al espacio disponible. 


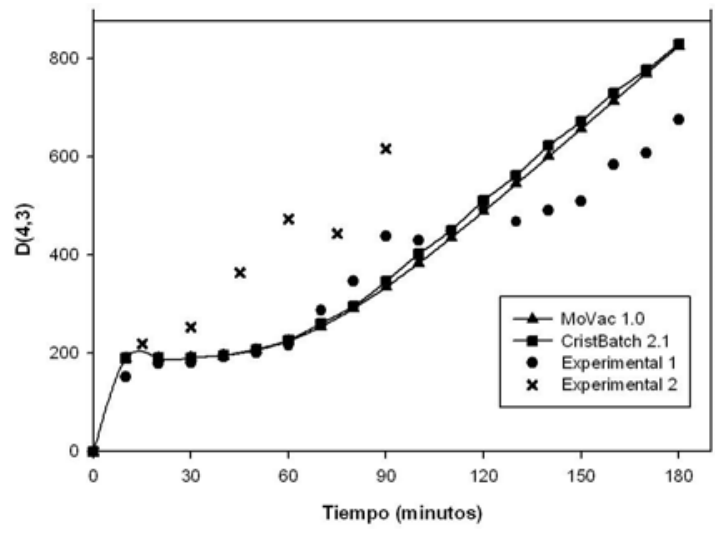

(a)

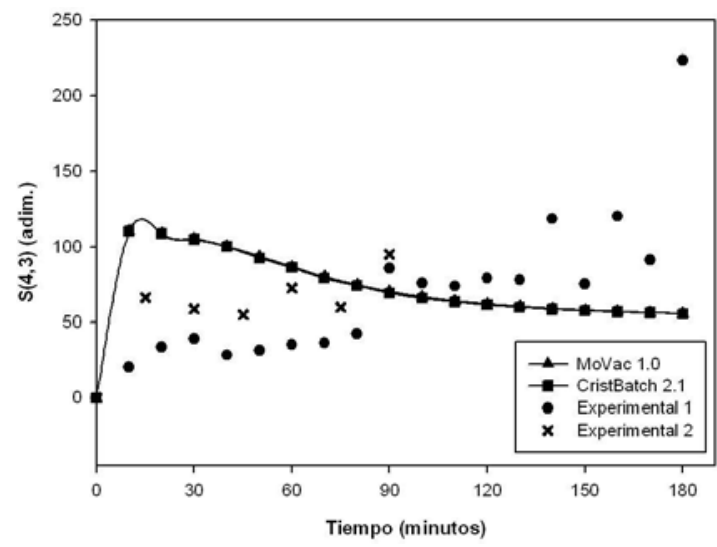

(b)

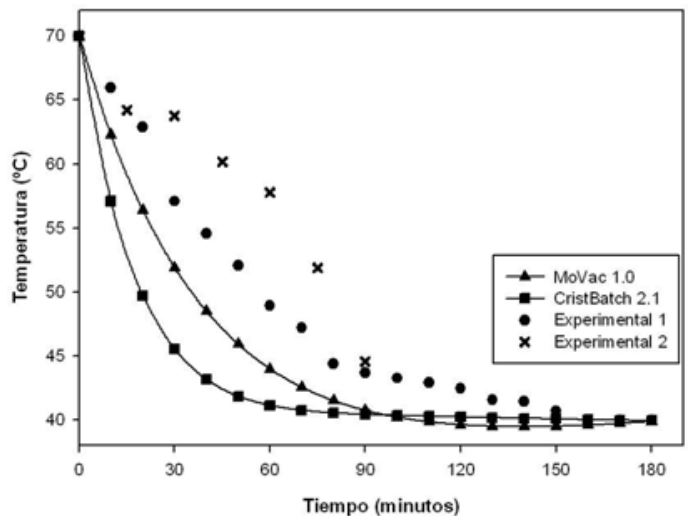

(c)

Fig. 7: Evaluación de resultados. (a) Diámetro promedio $(\mathrm{D}(4,3))$, (b) Desviación estándar $(\mathrm{S}(4,3))$, (c) Temperatura del interior del cristalizador

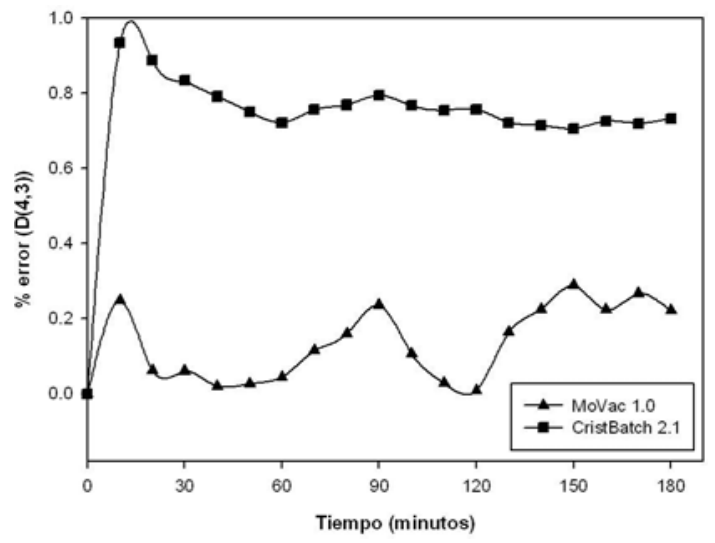

(a)

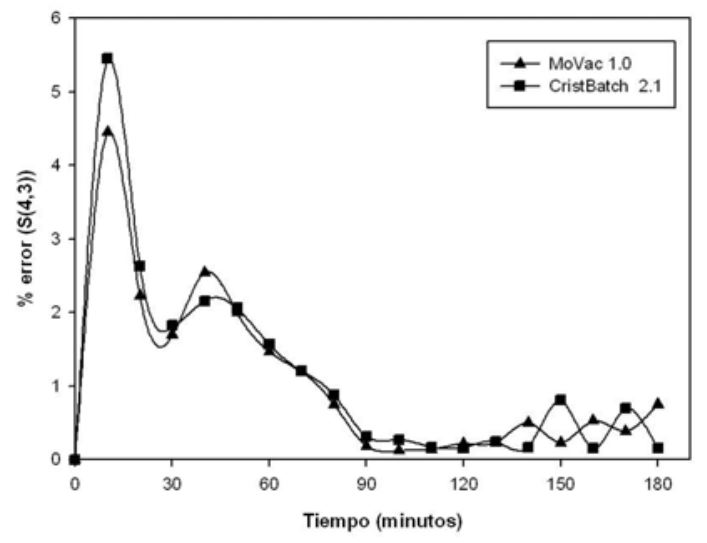

(b)

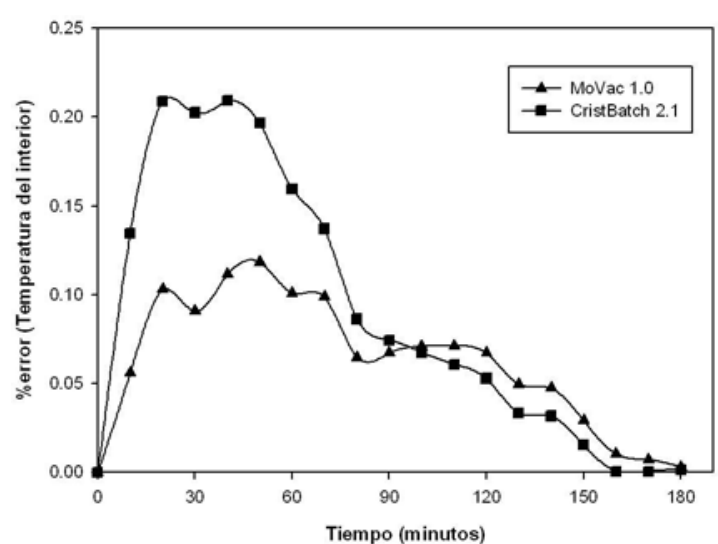

(c)

Fig. 8: Errores Relativos. (a) Diámetro promedio $(\mathrm{D}(4,3))$, (b) Desviación estándar $(\mathrm{S}(4,3))$, (c) Temperatura del interior del cristalizador. 


\section{CONCLUSIONES}

El simulador logra resultados numéricos satisfactorios fácilmente manipulables, que permiten visualizar la solución del modelo matemático de la cristalización batch a vacío, presenta una interfaz amigable al usuario, con la flexibilidad para cambiar los valores numéricos de las variables y parámetros, facilitando la comprensión del sistema en estudio.

Los resultados obtenidos por medio del simulador MoVac 1.0 son comparables con los obtenidos por otros simuladores del proceso de cristalización. También son comparables con resultados obtenidos mediante la experimentación en equipo piloto a vacío y por enfriamiento.

Los resultados permiten vislumbrar la aplicación del simulador para obtener ciertas características de los cristales formados esperados, implementando determinadas condiciones de operación y condiciones iniciales alimentadas al simulador.

\section{AGRADECIMIENTOS}

El desarrollo del trabajo estuvo soportado parcialmente a través del proyecto CONACYT FOMIX Veracruz 41691.

\section{NOMENCLATURA}

Letras latinas mayúsculas

\begin{tabular}{|c|c|c|}
\hline$A$ & Área total de transferencia de calor & $\mathrm{cm}^{2}$ \\
\hline$B$ & Velocidad de nucleación & Núm. part. $\bullet\left(\mathrm{cm}^{-3}\right) \cdot\left(\mathrm{min}^{-1}\right)$ \\
\hline$B(L)$ & Función distribuida de nacimientos & - \\
\hline C & Concentración del soluto & g de azúcar $\bullet g^{-1}$ de agua \\
\hline$C p$ & Capacidad calorífica & $\mathrm{cal} \cdot\left(\mathrm{g}^{-1}\right) \cdot\left({ }^{\circ} \mathrm{C}^{-1}\right)$ \\
\hline$D(L)$ & Velocidad de muerte & Núm. part. $\bullet\left(\mathrm{cm}^{-3}\right)\left(\mathrm{min}^{-1}\right)$ \\
\hline$D(m, n)$ & Distribución de momentos & - \\
\hline$F$ & Flujo másico & $g \cdot \min ^{-1}$ \\
\hline FTM & Factor de transformación de la malla & $\mathrm{cm}$ \\
\hline G & Velocidad de crecimiento & $\mathrm{cm} \cdot \min ^{-1}$ \\
\hline$K_{b}$ & $\begin{array}{l}\text { Constante de la velocidad de } \\
\text { nucleación }\end{array}$ & Núm part. $/\left(\mathrm{cm}^{-3}\right)\left(\mathrm{min}^{-1}\right)\left(\mathrm{g} \cdot \mathrm{cm}^{-3}\right)^{-\mathrm{j}}$.(r.p.m. $)^{-\mathrm{p}}$ \\
\hline$K_{v}$ & Factor de forma del cristal & adim \\
\hline$L$ & $\begin{array}{l}\text { Longitud, tamaño del núcleo, diámetro } \\
\text { del núcleo }\end{array}$ & $\mathrm{cm}$ \\
\hline M & Masa & $\mathrm{g}$ \\
\hline$N$ & $\begin{array}{l}\text { Velocidad de agitación, número de } \\
\text { partículas por unidad de volumen }\end{array}$ & $(\mathrm{rev})\left(\min ^{-1}\right)$ \\
\hline$P$ & Presión & inHg \\
\hline $\mathrm{Sr}$ & Sobresaturación relativa & adim \\
\hline$T$ & Temperatura & ${ }^{\circ} \mathrm{C}$ \\
\hline$U$ & Coeficiente de transferencia de calor & cal $\cdot\left({ }^{\circ} \mathrm{C}^{-1}\right)\left(\mathrm{min}^{-1}\right)\left(\mathrm{cm}^{-2}\right)$ \\
\hline$V$ & Volumen & $\mathrm{cm}^{3}$ \\
\hline$W$ & Velocidad de la corriente de vapor & $g \cdot \min ^{-1}$ \\
\hline
\end{tabular}

Letras latinas minúsculas

$\begin{array}{lll}n & \begin{array}{l}\text { Número de unidades elementales, } \\ \text { densidad de población (número) }\end{array} & \text { Núm. part. } \bullet\left(\mathrm{cm}^{-3}\right)\left(\mathrm{cm}^{-1}\right) \\ t & \text { Tiempo } & \text { min }\end{array}$


Letras griegas

\begin{tabular}{|c|c|c|}
\hline$\Delta H_{c}$ & Entalpía de cristalización del licor & cal•g ${ }^{-1}$ \\
\hline$\Delta L$ & Intervalo de longitud del cristal & $\mathrm{cm}$ \\
\hline$\mu$ & $\begin{array}{l}\text { Momento de distribución (volumen, } \\
\text { superficie, longitud, número) }\end{array}$ & \\
\hline & Densidad del crisal & $\cdot \mathrm{cm}^{-3}$ \\
\hline
\end{tabular}

Subíndices

$\begin{array}{ll}\text { c } & \text { Condensado } \\ \text { ev } & \text { Evaporado } \\ g & \text { Exponente de la constante de la } \\ & \text { velocidad de crecimiento } \\ j & \text { Chaqueta de calentamiento } \\ \text { s } & \text { Vapor (steam) } \\ \text { sat } & \text { Saturación } \\ T & \text { Total }\end{array}$

Superíndices

b Exponente de la sobresaturación en la velocidad de nucleación

$g \quad$ Exponente de la sobresaturación en la velocidad de crecimiento

j $\quad$ Exponente de la masa total en la velocidad de nucleación

p Exponente de la velocidad de agitación en la velocidad de nucleación

$q \quad$ Exponente de la velocidad de agitación en la velocidad de crecimiento

$r \quad$ Exponente de la velocidad de agitación en la velocidad de producciónreducción, orden de reacción

\section{REFERENCIAS}

Carrillo A.J.; "Diseño y simulación de estrategias para el control no lineal de la sobresaturación en la cristalización por lotes a través de perfiles dinámicos de regulación de vacío y estimación de parámetros". Tesis de Maestría en Ciencias en Ingeniería Química. I. T. de Orizaba. Mexico (2006).

Gerstlauer A.S., A. Motz y A. Mitrovic; "Development, Analysis and Validation of Population Models for continuous and batch crystallizer". AIChE J.: 8(5), 1123-1126 (2002).

Laloue N. y otros cinco autores; Dynamic Modeling of a Batch Crystallization Process: A Stochastic Approach for Agglomeration and Attrition Process, Chem. Eng. Science: 62(23), 6604-6614 (2007).

Martins P.M., F.A. Rocha y P. Rein; Modeling Sucrose Evaporative Crystallization. Part 1. Vacuum Pan Monitoring by Mass Balance and Image Analysis Methods, Ind. Eng. Chem. Res.: 44, 8858-8864 (2005a).

Martins P.M., F.A. Rocha y P. Rein; Modeling Sucrose Evaporative Crystallization. Part 2. Investigation into Crystal Growth Kinetics and Solubility, Ind. Eng. Chem. Res.: 44, 8865-8872 (2005b).

Mullin, John W.; Crystallization (4th ed.)., Oxford, UK: Butterworth-Heinemann, (2001) 
Perry R.H. y D.W. Green; Manual del Ingeniero Químico. McGraw-Hill, 7ª Edición, 2003.

Ulrich J.; Solution Crystallization-Developments and New Trends, Chem. Eng. Technol.: 26(8), 832-835 (2003).

Vollmer U. y J. Raisch; Population Balance Modelling and $\mathrm{H}$-Controller Design for a Crystallization Process. Chem. Eng. Science: 57, 4401-4414 (2002).

Wulkow M., A. Gerstlauer y U. Nieken; Modeling and Simulation of Crystallization Processes Using PARSIVAL (Particle Size Evaluation), Chem. Eng. Science: 56, 2575-2588 (2001).

Yang A., X. Ou, G.A. Montague y E.B. Martin, Local approximation to complex models for efficientoptimisation: application to crystallisation processes. Proceedings of European Congress of Chemical Engineering-ECCE-6 (2007). http://www.nt.ntnu.no/users/skoge/prost/proceedings/ecce6 sep07/upload/1316.pdf (11/01/08). 